\title{
Programa escolar en educación secundaria con menores infractores
}

Teaching School Program in Secondary Education with Juvenile Offenders

Programa escolar no ensino médio com menores infratores

\author{
Nuria López-Roca* (iD orcid.org/ 0000-0003-1934-1801 \\ María Fernández-Hawrylak** iD orcid.org/0000-0002-7222-587X \\ Jesús Soldevila-Pérez*** iD orcid.org/0000-0002-3712-6825 \\ Joan Muntaner-Guasp**** (iD orcid.org/0000-0002-2485-3257
}

Para citar este artículo: López-Roca, N., Fernández-Hawrylak, M., Soldevila-Pérez, J. y Muntaner-Guasp, J. (2021). Programa escolar en educación secundaria con menores infractores. Revista Colombiana de Educación, /(82), 15-36. https://doi.org/ 10.17227/rce.num82-10340

\begin{tabular}{lr}
\hline (c) $\$$ NC & Recibido: 24/07/2019 \\
Evaluado: 02/09/2020
\end{tabular}

* $\quad$ Licenciada en Ciencias de la Educación. Directora IES Can Balo de Palma de Mallorca, (España). Profesora Asociada en la Universidad de las Islas Baleares. Correo: nurialopez@iescanbalo.com

** Doctora. Profesora Titular de la Universidad de Burgos, Facultad de Educación. Correo: mfernandez@ubu.es

*** Doctor con mención internacional. Profesor de la Universidad de Vic-Central de Cataluña. Correo: jesus.soldevila@uvic.cat

**** Doctor en Pedagogía. Catedrático de Didáctica y Organización Escolar, Universidad de las Islas Baleares, Facultad de Educación. Correo: joanjordi.muntaner@uib.es 


\section{Resumen}

La educación junto con la familia son pilares fundamentales en los procesos de intervención de quienes han cometido delitos y por ello han salido prematuramente del sistema educativo ordinario. En este artículo se pretende describir el programa educativo que lleva a cabo un equipo del Instituto de Enseñanza Secundaria de Palma de Mallorca (España), el cual, por medio de una labor continuada de dotación de herramientas educativas, sociales y emocionales, busca la reincorporación al contexto escolar y social de referencia, de menores/jóvenes infractores que tienen que cumplir medidas judiciales de internamiento firme o cautelar. Se trata de un estudio cuantitativo, observacional y descriptivo. La satisfacción de los menores y de los padres con el programa, pone de manifiesto el éxito educativo de este alumnado, tanto desde el punto de vista de la escolarización como de la promoción, siendo los menores y sus familias quienes al finalizar el proceso valoran positivamente esta propuesta. Se concluye que la educación debe abandonar los condicionantes asistenciales, terapéuticos y compensatorios que la sitúan en la perspectiva del fracaso personal, y abrirse a propuestas que contengan una visión relativa del éxito educativo adecuada a cada uno de los alumnos para empoderarles hacia posturas de autoestima y empatía hacia su propio proceso formativo. El término éxito debe ser interpretado desde una perspectiva global y no unido al concepto de rendimiento académico.

\section{Palabras clave}

Inclusión; integración escolar; delincuencia juvenil; intervención; educación secundaria; formación profesional

\section{Keywords}

Inclusion; school desegregation; juvenile delinquency; intervention; secondary education; vocational education.

\begin{abstract}
Education is, along with family, the essential pillar in the processes of those who have committed offences a nd therefore have I eft p rematurely the o rdinary educational system. In this text, we describe the teaching program that the educational team of a high school in Palma de Mallorca (Spain) is developing, in order to reincorporate those juvenile offenders who have to serve judicial final or precautionary measures confinement to the school in social reference contexts. A continuous effort is made inside the Program, to provide educational, social and emotional tools. We carried out a quantitative, observational and descriptive study where the minors and their parents' satisfaction with the program, highlights the educational success of these students, both from the point of view of schooling and of promotion. In addition, minors and their families value this proposal positively. Education must abandon the supporting, therapeutic and compensatory determinants that place it in a perspective of personal failure, where it should be open to proposals that contain a relative vision of educational success appropriate for each of the students, in order to empower them towards positions of self-esteem and empathy on their own training process. The term success must be understood from a global perspective and should not be linked to the concept of academic performance.
\end{abstract}

\section{Resumo}

A educação é, juntamente com a família, o pilar fundamental nos processos de intervenção de quem cometeu crimes e, portanto, abandonou prematuramente o sistema escolar normal. Neste artigo descrevemos o programa educativo realizado pela equipa pedagógica do Instituto de Educação Secundária de Palma de Maiorca (Espanha), que visa a reincorporação de menores/jovens delinquentes que devem cumprir medidas judiciais de internamento firme ou cautelar. Nele, um trabalho contínuo é feito para fornecer ferramentas educacionais, sociais e emocionais. Este foi um estudo quantitativo, observacional e descritivo. A satisfação dos jovens e os pais com o programa evidencia o sucesso educacional desses alunos tanto do ponto de vista de escolarização quanto de promoção. Além disso, os menores e seus familiares valorizam positivamente a proposta. Concluímos que a educação deve abandonar as condições assistenciais, terapêuticas e compensatórias que a colocam na perspectiva do fracasso pessoal e estar aberta a propostas que contenham uma visão relativa de sucesso educacional adequada a cada um dos alunos

N. ${ }^{\circ} 82$ para empoderá-los para posições de autoestima e empatia para com o seu próprio processo de formação. O termo sucesso deve ser interpretado de uma perspectiva global e não vinculado ao conceito de desempenho acadêmico.

\section{Palavras-chave}

Inclusão; integração escolar; delinquência juvenil; eficácia do programa; intervenção; ensino médio; formação profissional 


\section{Introducción}

La situación educativa de los menores y jóvenes infractores con medidas judiciales de privación de libertad requiere de actuaciones innovadoras que rompan con la dinámica negativa en su vida escolar, formativa y socio-familiar.

El objetivo de este artículo es describir un programa escolar pionero desarrollado en la educación secundaria con menores que deben cumplir medidas judiciales de internamiento firme o cautelar. El programa tiene como objetivo principal la reincorporación con perspectivas de éxito de las chicas y los chicos que, por razones judiciales, han salido prematuramente del sistema escolar. Este programa pretende mostrar que, a partir de un diseño de intervención global, estos alumnos infractores con medidas judiciales, privados de libertad, pueden volver con éxito no solo al circuito escolar ordinario, sino a sus contextos sociales como personas competentes en los diferentes ámbitos de su vida. Para ello, es necesario replantear determinadas creencias y actuaciones pedagógicas o punitivas que se creían acertadas y adecuadas, ya que ahora se demuestran insuficientes, inadecuadas y poco ajustadas a la realidad actual de la sociedad a la que sirven y del alumnado al que se quiere dar respuesta. Estos alumnos, que salieron del sistema escolar de manera prematura por diversas razones, que fueron catalogados como "extremadamente diversos" y que no encontraron respuestas favorecedoras a sus características y necesidades como alumnos y como personas en el sistema educativo, precisan de espacios escolares que les devuelvan la seguridad y la autoestima suficiente para trabajar en su desarrollo y en su propio aprendizaje, y les permitan de nuevo su incorporación y participación al mundo escolar y social.

Esta propuesta comparte la idea que avalan ya desde 1990 las Directrices de las Naciones Unidas para la Prevención de la Delincuencia Juvenil (Resolución 45/112) y recientes estudios (Luna et ál., 2018; Mampaso et ál., 2014; Pérez, 2017; Poch y Zaplana, 2017) que señalan que uno de los principales objetivos de la intervención con menores infractores debe ser mejorar su nivel educativo y social. Además, recogen que el mejoramiento cultural y educativo es un factor necesario de gran relevancia preventiva. Así, pues, los tres pilares claves en que se fundamenta la propuesta son: la educación inclusiva, el concepto relativo de éxito y el planteamiento integral de la intervención didáctica. 


\section{La educación inclusiva}

Históricamente, como señalan Palacios y Romañach (2006), la diferencia en el ámbito educativo se interpreta en la escuela desde la perspectiva del déficit, donde lo que predomina en la observación y la definición del alumno son las deficiencias y las dificultades que presenta en su proceso de aprendizaje, lo cual lo aleja de la norma y lo hace aparecer como distinto e inferior al resto de sus compañeros. Esta perspectiva, propia de la Educación Especial y del paradigma de la integración, se caracteriza por: 1) fundamentar todas sus intervenciones en las limitaciones del alumno; 2) pensar que el problema está en el alumno, que presenta déficits que le impiden o dificultan su aprendizaje y lo hacen distinto; y 3) promover la terapia individual como mecanismo básico de intervención para ayudar a superar estos déficits con el objetivo de asimilarlos a la normalidad (Marchesi, 2014; Parrilla, 2002).

Frente a esta posición, que conduce a la segregación y a la discriminación del alumnado por sus diferencias en la escuela ordinaria, se desarrolla el modelo de la educación inclusiva (Arnaiz, 2019; Echeita y Ainscow, 2011; Muntaner et ál., 2016, Organización de las Naciones Unidas para la Educación, la Ciencia y la Cultura-Unesco, 2016, 2017), que se basa en: 1) modificar el contexto y las oportunidades que se ofrecen a todos los alumnos para que puedan participar y aprender en entornos ordinarios y normalizados con el resto de sus compañeros; 2) aceptar la diversidad como un componente inevitable con el que se debe aprender a trabajar como un elemento enriquecedor, nunca reducirlo ni eliminarlo; y 3) ofrecer la educación como modelo de intervención en todos los alumnos, sustituyendo la terapia y dando relevancia a las propuestas formativas y de aprendizaje en igualdad y equidad. Este planteamiento permite superar el modelo individual de intervención para centrarse en el modelo curricular (Ainscow, 1995).

El modelo de la educación inclusiva se basa en ofrecer respuestas de calidad a la diversidad real del alumnado desde un planteamiento equitativo, no basado en enfoques asistenciales, compensatorios, punitivos o focalizados, sino con un fuerte carácter preventivo y global. Este es el gran desafío al que se enfrentan los centros educativos y los docentes en la actualidad. Como afirma Blanco: "este modelo exige cambios sustantivos en las concepciones, actitudes, el currículo, las prácticas pedagógicas, la formación de los docentes, los sistemas de evaluación y la organización de las escuelas" (2008, p. 8). Estos cambios nos Ilevan a repensar constantemente las prácticas pedagógicas de los centros educativos, con la finalidad de aumentar su capacidad para responder de manera adecuada a la diversidad existente en la actualidad (Ainscow y Messiou, 2018). 
El marco de referencia de la educación inclusiva está marcado por un proceso que se plantea a partir de tres premisas (Booth y Ainscow, 2002):

» Identificar las barreras que, desde distintos planos de la vida escolar, interactúan negativamente con las condiciones personales o sociales del alumno, que limitan su actividad general en el centro y en el aula.

» Planificar, implementar y sostener procesos de innovación y mejora escolar que permitan superar estas barreras, favoreciendo y facilitando el aprendizaje de todos los alumnos en la dinámica general del centro y del aula.

» Reestructurar las culturas, las políticas y las prácticas escolares existentes, de forma que progresivamente respondan con más equidad a la diversidad del alumnado.

Este planteamiento propone el desarrollo de dos ideas: por una parte, la inclusión educativa se define (Ainscow et ál., 2006; Echeita et ál., 2009) por la presencia, la participación y el aprendizaje de todos los alumnos en su grupo escolar de referencia. Por otra parte, el proceso de cambio hacia una educación inclusiva abarca tres niveles de actuación, que pueden apoyar o perjudicar los avances de los proyectos inclusivos para influir directamente en los procesos de enseñanza y aprendizaje (Booth y Ainscow, 2002). Estos se tuvieron en alta consideración en el diseño y la creación del programa que se presenta: creación de culturas inclusivas, elaboración de políticas inclusivas y desarrollo de prácticas inclusivas.

Para Wehmeyer (2009), las prácticas inclusivas deben poner el énfasis en la calidad del programa educativo que se aplica para asegurar intervenciones que proporcionen alta calidad educativa para todos los alumnos sin excepciones y altas cotas de éxito.

\section{Concepto relativo de éxito}

La palabra éxito se refiere a un resultado final y satisfactorio de una tarea o proceso. Esta definición plantea tres aspectos que se deben tener en cuenta (Fandiño, 2008): 1) cumplimiento de metas y objetivos; 2) fin o terminación de procesos; 3 ) satisfacción y respuesta a deseos o necesidades profesionales, económicas, sociales y personales. Así, al hablar de éxito, no necesariamente se hace referencia a triunfos en la vida, sino al alcance de "logros integrales", entendidos como la consecución de metas y objetivos en varias esferas de la vida, no solo en una (Fandiño, 2008). Se trata de un concepto subjetivo, condicionado a las características de las propias tareas y a las circunstancias de los sujetos que las realizan. 
En el contexto escolar, el concepto de éxito se vincula al de rendimiento académico, que normalmente se mide a partir de la evaluación numérica de los resultados de pruebas estandarizadas; o bien, ligado a la promoción académica de los diferentes cursos que componen las distintas etapas educativas que marca el propio sistema (Edel, 2003; Feito, 2009). Esta es una visión sesgada, ya que una propuesta educativa dirigida a la formación y el desarrollo integral de la persona exige contextualizar claramente la manera de entender el éxito, que no solo se relaciona con los resultados académicos finales, sino con el proceso de aprendizaje y de formación asumido en cada momento por cada persona. Supone adoptar otro tipo de pensamiento, y una postura crítica justamente contraria a la teoría y la práctica educativas basadas en la selección, la competición y el individualismo (Barton, 2009).

Los alumnos protagonistas de este trabajo - menores infractoresson categorizados en el marco de la escuela selectiva y tradicional con etiquetas tan peyorativas y ofensivas como: alumnos terminales, alumnos con problemas graves de conducta, delincuentes, etc. La consecuencia de este rechazo conduce a la exclusión prematura del sistema educativo, pero además conlleva la interiorización de un fracaso continuo, que se entiende como personal y se acompaña de una inadaptación a las condiciones rígidas del propio sistema (Poch y Zaplana, 2017).

Esta realidad nos sitúa ante una pregunta clave expuesta por De Andrés y Fernández-Hawrylak: “¿Podemos considerar como éxito que un alumno que encuentra dificultades académicas consiga finalizar un programa o etapa educativa?" (2015, p. 114). La respuesta evidente es que sí. El éxito académico es, en todos los casos, avanzar hacia caminos de inclusión escolar y social, y se traduce en prácticas que promueven su progreso como persona competente, en experiencias formativas y personales constructivas y —más concretamente en el caso que nos ocupa- en alejarse del ámbito de la delincuencia. En la educación inclusiva "la consecución de un objetivo curricular concreto no siempre es la única, ni tan siquiera la principal, meta para garantizar el éxito y la felicidad en la vida" (Stainback et ál., 1999, p. 85).

Por ello, uno de los objetivos planteados es entender el concepto de éxito desde una perspectiva abierta, global y, sobre todo, adaptada al perfil de los protagonistas. Este éxito dependerá, al mismo tiempo, de los itinerarios formativos que realicen, las expectativas que se planteen, y el acompañamiento continuo y adaptado que se aplique en cada momento del proceso. Desde esta forma de conceptualizarlo, el éxito educativo supone garantizar un desarrollo óptimo de la persona, que le posibilite un retorno a la comunidad escolar y social. 


\section{Planteamiento integral de la propuesta didáctica}

El éxito de que un menor infractor vuelva a ser un alumno competente académica y socialmente no dependerá solo de la intervención particular que se haga con él, sino de la necesidad de asumir responsabilidades de manera compartida entre todos los agentes implicados como única vía para alcanzar los objetivos marcados. Así lo justifican Fernández-Campoy et ál. (2017) cuando señalan que la reinserción social y laboral de los menores infractores, al configurarse como un proceso muy ambicioso y complejo, para resultar verdaderamente efectiva, requiere de un exhaustivo mecanismo de planificación, de coordinación, de estructuración, y de un trabajo interdisciplinar que implica una coordinación interinstitucional importante.

En consecuencia, si se quiere conseguir un desarrollo global óptimo para cada uno de estos menores, debe haber un trabajo coordinado entre todos los profesionales implicados para conseguir actuaciones cohesionadas y coherentes que puedan dar como resultado una intervención integral eficaz que se traducirá en procesos de desarrollo del éxito.

Este trabajo en red debe implementarse también en la propuesta educativa desarrollada en la época de internamiento, que se basa, de manera general, en los siguientes aspectos:

»El reconocimiento explícito de la heterogeneidad de los participantes en el proceso de enseñanza-aprendizaje, partiendo de un currículo común que se adapta a las distintas características del alumnado que configura un grupo. Como escribe Gimeno: "la escuela como institución tiene ante sí la necesidad de articular en su estructura y en su funcionamiento el derecho a la igualdad y el derecho al respeto a la singularidad" (2000, p. 47).

» Un modelo contextual. Si se parte de la premisa de que el problema no es el alumnado, se debe plantear una reorganización del contexto, de modo que sea este el que se adapte a sus características y necesidades. Un contexto que sea acogedor, con espacios abiertos y flexibles donde se puedan compartir experiencias y actividades, y donde el alumnado sienta la necesidad de formación y se sientan también protagonistas de su aprendizaje (Sapon-Shevin, 2013).

» Diversificación y flexibilización de los recursos, las estrategias metodológicas, los tiempos y los espacios de aprendizaje. Un aspecto importante por considerar es la relación inversa que existe entre el nivel de conocimientos previo del alumnado y la cantidad y calidad de la ayuda educativa necesaria para lograr los objetivos. De acuerdo con esta idea, el alumnado con menos conocimientos previos requerirá métodos y recursos que impliquen un alto grado de ayuda, 
mientras que el alumnado con más conocimientos previos podrá aprender a partir de planteamientos metodológicos y recursos que impliquen una mayor autonomía (Echeita, 2019).

» El trabajo colaborativo y el aprendizaje cooperativo. Este programa pone atención a estos dos aspectos: la imprescindible colaboración entre todo el equipo docente, donde se trabaja desde la ayuda, la suma de esfuerzos, el debate, la coordinación, el acuerdo y la comunidad; y el desarrollo de estructuras de aprendizaje que establecen relaciones cooperativas entre el alumnado, organizando las actividades a partir de situaciones donde es necesario interactuar para adquirir nuevos conocimientos. Este tipo de estructura crea interdependencia positiva entre el alumnado y potencia la responsabilidad individual y grupal, hecho que enriquece los procesos de aprendizaje (Pujolàs, 2008; Pujolàs et ál., 2013).

\section{Programa escolar con menores infractores}

El trabajo que se presenta se contextualiza en las quince unidades docentes de un Instituto de Enseñanza Secundaria de Palma de Mallorca, vinculado a través de un convenio a la Fundación Instituto S'Estel (FISE), organismo encargado en las Islas Baleares de la gestión de los centros que ejecutan medidas de privación de libertad marcadas por la Ley Orgánica 5/2000, reguladora de la Responsabilidad Penal de los Menores (LORPM), y que depende de la Consejería de Asuntos Sociales y Cooperación, Dirección General de Menores y Familia del Gobierno Balear. Se trata de una fundación de iniciativa pública que tiene como finalidad atender educativa y socialmente a los usuarios y a sus familias, en cooperación, colaboración y coordinación con los recursos y servicios comunitarios del entorno social que le son propios.

Actualmente cuenta con tres centros socioeducativos: Es Pinaret (con ocho aulas), Es Fusteret (con dos aulas) y Es Mussol (con un aula). Además, dispone de un espacio externo, el Edificio вов (con dos aulas), donde se imparte el primer curso de tres grados de Formación Profesional Básica (Agrojardinería y Composiciones Florales, Informática y Comunicaciones, y Cocina y Restauración) a menores con proceso educativo positivo como medida puente (los siguientes cursos se imparten en otros centros o institutos de la Comunidad, en función del domicilio familiar del menor, con el compromiso de acceder a una plaza en el grado iniciado).

En estas aulas, anteriormente denominadas unidades docentes externas, se imparten las modalidades educativas que reflejan los textos de la normativa vigente para alumnado mayor de catorce años (Enseñanza Secundaria Obligatoria-ESO y Enseñanza Secundaria para Adultos-ESPA, 
Bachillerato-BACH y Formación Profesional Básica-FPB, además de la preparación para las pruebas de acceso a Grado Medio y Superior de Formación Profesional y Graduado Escolar, de pruebas libres — se prepara, no se evalúa-, así como alfabetización, usualmente para alumnado inmigrante - hasta que está preparado para acceder a primer curso de la ESPA—) a través de un convenio prorrogado de manera regular cada curso escolar con la Consejería de Asuntos Sociales y Cooperación-Dirección General de Menores y Familia y la Consejería de Educación y Universidad-Dirección General de Comunidad Educativa e Innovación (el último convenio se suscribió el 22 de enero del 2015).

Cada unidad posee una idiosincrasia propia por las características del centro socioeducativo donde está ubicada y por el perfil del alumnado al que atiende. La mayoría de ellas son unitarias, concentran alumnado de diferentes niveles educativos y edades y pueden tener diferentes horarios, modalidades o, incluso, distintos tiempos en los procesos de retorno al centro escolar de referencia (Instrucción, 20/18, de 1 de septiembre).

Hoy estas unidades se configuran como la entidad virtual Instituto de Enseñanza Secundaria Can Balo (pionero en España) a través del Decreto 25/2017, de 19 de mayo. Su finalidad es fomentar el desarrollo integral de los menores que se encuentran cumpliendo medidas judiciales, mediante estrategias educativas, sociales y emocionales que favorezcan su reincorporación formativa y laboral, así como una transición constructiva y positiva hacia la vida adulta. En la actualidad, dispone de un equipo docente formado por 18 profesores pertenecientes a la Consejería de Educación y Universidad. A ello se une un equipo técnico compuesto por trabajadores sociales, psicólogos, pedagogos y educadores sociales que dependen de la Fundación Instituto Educativo S'Estel FISE, y subcontratas para personal de cocina, de limpieza y cuerpo de seguridad. La FISE, en su conjunto, tiene una capacidad para 100-110 alumnos con medidas judiciales de privación de libertad, que a su vez son los estudiantes atendidos por el equipo docente del IES Can Balo. El IES acoge a estudiantes de entre 14 y 21 años (algunos ya no son menores, pero delinquieron antes de cumplir la mayoría de edad) que tienen que cumplir una medida judicial de internamiento firme o cautelar en régimen abierto, semiabierto o cerrado.

Tras la experiencia piloto del curso 2017/2018, en el actual se ha abierto una unidad docente en la Asociación Proyecto Hombre Baleares (sección Proyecto Joven), para alumnado con medidas judiciales de privación de libertad que, además, debe formar parte de un programa de deshabituación. Esta aula desarrolla la modalidad de ESPA y se presenta como un recurso que complementa la intervención terapéutica, dando importancia a la formación académica como factor de protección, prevención y elemento facilitador de su reinserción social. 
Dentro de su misión, la FISE incluye el compromiso de mejora continua en su sistema de gestión y se responsabiliza de cumplir con los requisitos legales correspondientes a sus características y actividades. Su visión se basa en ser una organización distinguida y de referencia para prevenir y abordar las diferentes situaciones sociales de los usuarios desde un enfoque educativo y multidisciplinar, y dotarlos de recursos para afrontar la vida cotidiana de una manera prosocial y competente. Todas estas aulas están regidas por el programa educativo que se presenta en este artículo.

\section{Perfil del programa escolar que se aplica}

El programa escolar de las unidades docentes adscritas a la Fundación S'Estel se empezó a gestar en 1999 y ha ido creciendo, ampliándose y actualizándose durante diecinueve cursos escolares con la finalidad de mejorar la atención escolar y formativa de los menores internados, garantizar una educación de calidad para todos ellos, y constituir una herramienta de inserción social y escolar. Desde el Programa de Enseñanza Reglada (PER) se busca promover una enseñanza pública y aconfesional de calidad, que valore la diversidad en todas sus manifestaciones (cultural, ideológica, religiosa, sexual, económica, entre otras). Por tanto, las unidades docentes dan respuesta a la diversidad del alumnado, teniendo en cuenta sus conocimientos previos, capacidades, situación judicial e intereses personales y sociales. Asimismo, se favorece el contacto del alumnado con las características definitorias del país en general y de la comunidad autónoma en particular, y se fomenta el conocimiento del patrimonio cultural y del entorno natural.

Los principios rectores relacionados con estos valores rigen las diferentes actuaciones, diseños de intervenciones o prácticas educativas resultado de la ejecución del programa:

» Favorecer la autonomía del alumnado

»Potenciar la cooperación entre el grupo

» Fomentar el uso de las tecnologías de la información y de la comunicación

» Emplear metodologías inclusivas (favorecedoras de la interacción, de la creación, de la metacognición y del compromiso)

» Impulsar una comunidad educativa abierta a la red social

» Partir de las capacidades y de las fortalezas de cada estudiante; promover la buena convivencia

» Proporcionar atención real y de calidad a la diversidad del alumnado

» Asumir la diversidad como fuente de riqueza, potenciando grupos heterogéneos para seguir una línea de cooperación y colaboración. 


\section{Objetivos y actuaciones}

El objetivo general del PER es promover, desde un abordaje individual, la educación integral de los jóvenes (que además son alumnos), proporcionándoles las herramientas que les ayuden a desarrollar sus competencias formativas, sociales, emocionales y de la vida cotidiana, necesarias para afrontar la realidad con los recursos personales apropiados, y posibilitar una adecuada y exitosa reincorporación familiar, escolar y social, y con ello una transición positiva hacia la vida adulta.

La consecución de este ambicioso objetivo demanda dos tipos de actuaciones:

» Dirigidas al alumnado. Esta línea de actuación va encaminada a dotar al menor/joven de herramientas y estrategias para su reincorporación escolar a través del desarrollo de sus capacidades, tratando de prepararlo hacia un retorno con éxito.

»Dirigidas al contexto. Desde esta línea se trabaja de manera directa con el entorno social, escolar (centro escolar de referencia) y familiar al cual el alumno va a volver. El trabajo con la familia va dirigido a reorganizar y reajustar el contexto familiar a través del Programa de Intervención Familiar (PIF) (López et ál., 2018), conscientes de que la implicación familiar tiene una incidencia directa en el rendimiento y el comportamiento.

\section{Principios metodológicos}

La metodología del PER se fundamenta en una perspectiva abierta y flexible, preparada para cambios o modificaciones en cualquier momento que sea necesario, con la finalidad de ir adaptándose a las necesidades de las diferentes unidades docentes, tanto en el conjunto del grupo clase como con cada uno de los alumnos. Esta flexibilidad obedece a que los menores/ jóvenes entran y salen de los centros en función tanto de su reincorporación escolar o laboral, como de la medida judicial, pero también por la diversidad de capacidades, intereses, edades, situaciones personales o centros escolares de referencia, lo que significa que el alumnado del grupo varía considerablemente de una semana a otra.

Otro factor que hay que tener en cuenta es que el perfil del alumnado, por lo general, coincide en algunos aspectos, como fracaso escolar, absentismo, rechazo hacia el mundo escolar, capacidades académicas mínimas o falta de habilidades sociales, por lo que el plan de intervención se desarrolla a partir de estas premisas. 
Para adaptarse a la diversidad de actitudes y capacidades que presenta el alumnado se tienen en cuenta unas prácticas educativas y principios metodológicos que impulsan el desarrollo del principio de inclusión o inserción del alumnado en el contexto democrático de procedencia: globalización, normalización, proximidad, equidad, participación, eficacia y eficiencia, coordinación, diseño universal de aprendizaje, y principios de sensibilización y de prevención, a través de la adquisición de hábitos de responsabilidad, habilidades sociales, tareas ejecutivas y educación emocional (Decreto 39/2011, de 29 de abril).

\section{Protocolo de acogida y proceso de reinserción}

Al día siguiente de su internamiento, el menor acude al aula en función de la modalidad educativa que le corresponde según su perfil (necesidades educativas, trayectoria escolar, habilidades, capacidades, etc.), a un espacio con características específicas con contención física propia de la estructura de los centros, hasta cuando pueda asumir el compromiso y el nivel de autonomía suficiente para asistir al centro de referencia. Dicha modalidad se refleja en el apartado de formación de su Programa Individual de Ejecución (PIE), que es comunicado al juzgado correspondiente para su aprobación (Instrucción 20/18, de 1. ${ }^{\circ}$ de septiembre).

La detección de necesidades y la búsqueda de información determinan la toma de decisiones para el diseño del apartado del PIE de cada menor relacionado con el ámbito formativo/la enseñanza oficial, teniendo en cuenta su edad, situación judicial (tipo de internamiento, régimen y duración de la medida), trayectoria formativa previa (análisis del historial académico e información curricular y comportamental en los anteriores centros escolares y recursos educativos disponibles), situación formativa en el momento de internamiento (matrícula activa, absentista intermitente, absentista crónico o sin matrícula activa), intereses a corto y mediano plazo en relación con la orientación vocacional, oferta formativa dentro y fuera de los centros en el momento de la valoración y de la elección de la actividad formativa y riesgo de reincidencia de la conducta delictiva por la que está internado.

A partir de los criterios marcados en las programaciones correspondientes al curso académico en el que el menor está escolarizado en su centro escolar de referencia, facilitadas por este, se diseña una propuesta curricular adaptada a sus necesidades y al contexto donde estará ubicado en el Centro correspondiente del IEs Can Balo. El profesorado de las unidades docentes concreta en cada aula la propuesta y la evalúa, mientras que el centro de referencia va homologando el proceso de evaluación y emitiendo los boletines de calificaciones correspondientes. 
Finalizada la medida judicial, los menores retornan a su entorno familiar, educativo y social, siempre que sea viable. La reincorporación escolar es uno de los aspectos más importantes a tener en cuenta desde el inicio de la medida de internamiento. Supone, por lo tanto, un objetivo que formará parte de la respuesta que se dará desde el IEs y que, además, se compartirá con el centro de referencia del menor para poder garantizar el derecho a una escolarización obligatoria integral y de calidad. Por ello, durante el inicio del internamiento se trabaja el proceso de reincorporación, procurando que sea lo más corto posible, pero real (según la situación personal y judicial del régimen) y tratando de que la reincorporación se produzca antes de que concluya el internamiento por las posibles recaídas (consumo, conducta, desadaptación al centro, ausencias, fugas, entre otras).

Los adolescentes que se enfrentan a circunstancias difíciles o que acumulan adversidades pueden necesitar un apoyo para hacer una evolución exitosa hacia la edad adulta, ya que los estudios han mostrado que los adolescentes en riesgo tienen más probabilidades de presentar una salud mental más deficiente, consumo de sustancias y comportamiento criminal (Garcia-Poole et ál., 2019).

Habitualmente, los programas de intervención para adolescentes se han dirigido a reducir comportamientos problemáticos individuales como el consumo de alcohol y drogas, violencia o riesgo sexual (Garcia-Poole et ál., 2019). Sin embargo, los programas de prevención universal ofrecidos en entornos formales durante la escuela o después del horario de clase a menudo no consiguen involucrar a los adolescentes que ya están experimentado fallas o abandono escolar, o han comenzado a mostrar un comportamiento problemático o delictivo (Kremer et ál., 2015).

Cuando la conducta del alumno hace evidente que está preparado para seguir la formación académica en su centro escolar de referencia y el contexto se ha reestructurado garantizando las herramientas necesarias para un retorno positivo, pasa a una modalidad combinada (semipresencial). A partir de este momento se trabaja de manera conjunta (profesorado de las unidades docentes y del centro escolar de referencia) para conseguir que se reincorpore en la modalidad presencial y que continúe su formación en dicho centro a tiempo completo. Mientras el alumno continúa con la medida de internamiento, se hace el seguimiento desde el IES Can Balo bajo la supervisión de un profesor asignado, al que se denomina profesor de referencia, para reajustar actuaciones ante conductas que supongan un retroceso en el proceso de reincorporación escolar. Antes de que finalice la medida de internamiento, este profesional hace un traspaso del caso al educador de medio abierto (EMA), quien será el responsable del seguimiento del proceso del alumno y el nuevo contacto con el centro escolar de referencia durante la fase de libertad vigilada. 


\section{Proceso de evaluación}

Como se ha indicado, en este contexto se entiende la evaluación como un proceso de doble vía: por un lado, la evaluación del menor para su retorno al contexto escolar de referencia; por el otro, la evaluación curricular que se desarrolla en su aula del IES, como en cualquiera de un centro escolar de la comunidad.

De la evaluación del proceso de reincorporación se deja constancia en las hojas de registro de las coordinaciones interinstitucionales, en las actas de reuniones y en el expediente del alumnado. Esta documentación va acompañada del acuerdo de la dirección del IES como último responsable del alumnado en dicho proceso.

Respecto a la evaluación curricular, esta se hace partiendo de las directrices marcadas por la Ley Orgánica 8/2013, para la Mejora de la Calidad Educativa (Lomce). Cada alumno es evaluado en función de la modalidad educativa de matrícula y materias o disciplinas que cursa. Tal y como marca la precitada ley, los criterios de evaluación y estándares de aprendizaje pactados por su centro escolar de referencia representan la hoja de ruta para poder garantizar una evaluación global y válida. Cabe añadir que las metodologías, recursos o herramientas de evaluación son variados y adaptados a las necesidades de cada perfil con el objetivo de asegurar resultados fiables y completos, pero siempre siguiendo las instrucciones de los textos legales referidos a qué enseñar y qué evaluar. La secuenciación es la misma que la del centro escolar de referencia, usualmente trimestral, o cuatrimestral en el caso de los estudios de ESPA y FPB. Si se produce el retorno durante ese periodo, la evaluación curricular será el resultado de una valoración conjunta entre los docentes del IES Can Balo y del centro escolar.

\section{Resultados de satisfacción con el programa escolar aplicado}

Al finalizar la medida de internamiento, las familias de los jóvenes responden un cuestionario de satisfacción confidencial, administrado por la Fundación S'Estel. Desde el curso 2017/2018, los menores que han finalizado las medidas judiciales también cumplimentan otro suministrado por el IES Can Balo. Ambos cuestionarios consisten en preguntas según una escala de tipo Likert de 5 puntos donde se especifica el nivel de acuerdo o desacuerdo con las afirmaciones presentadas (de $1=$ Nada satisfecho a 5 = Muy satisfecho), y espacios para comentarios personales. 
El cómputo de menores internados durante el curso 2017/2018 fue de 139, de los cuales 29 (20,86 \%) son mujeres y 110 (79,14\%) varones, entre 14 y 21 años $(M=16,58, D T=1,11)$ (tabla 1$)$, matriculados durante el internamiento tal como se recoge en la tabla 2. Antes de esta medida, el $61,87 \%$ se encontraba sin escolarizar (tabla 3 ).

Tabla 1.

Distribución de la muestra por edad y sexo

\begin{tabular}{cccc}
\hline Edad & Alumnos & Alumnas & Total \\
\hline 14 & 3 & 1 & 4 \\
\hline 15 & 6 & 5 & 11 \\
\hline 16 & 45 & 11 & 56 \\
\hline 17 & 38 & 8 & 46 \\
\hline 18 & 10 & 4 & 7 \\
\hline 19 & 7 & 0 & 0 \\
\hline 21 & 0 & 0 & 1 \\
\hline
\end{tabular}

Fuente: elaboración propia

La concentración de edad entre los 16 y 17 años podría deberse a la aplicación de medidas previas al internamiento, siendo esta la última en emplearse al ser la más restrictiva. También podemos ver por los datos de la tabla que las alumnas son más precoces en delinquir que los alumnos (el 20,68 \% tiene 15 años o menos, frente al 8,18\% de ellos), así como en reinsertarse (no hay ninguna alumna mayor de 18 años, mientras que sí hay 8 alumnos).

Tabla 2.

Nivel académico en el que se matricularon en el Centro Socioeducativo

\begin{tabular}{|c|c|c|c|}
\hline Nivel & Alumno & Alumna & Total \\
\hline ESO & 11 & 4 & 15 \\
\hline ESPA & 73 & 17 & 90 \\
\hline FP & 24 & 8 & 32 \\
\hline $\mathrm{BACH}$ & 2 & $\mathrm{O}$ & 2 \\
\hline Sin matrícula & $\mathrm{O}$ & $\mathrm{O}$ & $\mathrm{O}$ \\
\hline
\end{tabular}

Fuente: elaboración propia 
Tabla 3.

Nivel académico en el que estaban matriculados en el centro escolar de referencia

\begin{tabular}{|c|c|c|c|}
\hline Nivel & Alumnos & Alumnas & Total \\
\hline ESO & 17 & 9 & 26 \\
\hline ESPA & 12 & 5 & 17 \\
\hline FP & 4 & 4 & 8 \\
\hline BACH & 2 & $\mathrm{O}$ & 2 \\
\hline Sin matrícula & 75 & 11 & 86 \\
\hline
\end{tabular}

Fuente: elaboración propia

Las tablas 4 y 5 recogen los datos de promoción de curso del alumnado, considerada en función de la modalidad educativa. La reinserción se refiere exclusivamente a la escolar, no a la familiar y social. Como vemos, aunque previamente al internamiento la mayoría del alumnado $(61,87 \%)$ no estaba escolarizado, el 66,91 \% alcanza la promoción.

Tabla 4.

Promoción de los alumnos

\begin{tabular}{lcc}
\hline & Promocionan & No promocionan \\
\hline Reinsertados & 23 & 11 \\
\hline No reinsertados & 49 & 27 \\
\hline
\end{tabular}

Fuente: elaboración propia

Tabla 5.

Promoción de las alumnas

\begin{tabular}{lcc}
\hline & Promocionan & No promocionan \\
\hline Reinsertadas & 13 & 3 \\
\hline No reinsertadas & 8 & 5 \\
\hline
\end{tabular}

Fuente: elaboración propia

A 30 de junio del 2018, fecha de finalización del curso escolar, 78 menores continuaban con medidas de internamiento, mientras que 61 (49 alumnos y 12 alumnas) las habían terminado. De estos, 44 cumplimentaron el cuestionario sobre aspectos de las aulas donde habían estudiado durante el internamiento.

Los alumnos evalúan positivamente el desarrollo de las clases, destacando que el ítem mejor puntuado es "Valoro lo que he aprendido" $(M=4,36, D T=0,49)$. Respecto al acompañamiento de su profesor de 
referencia, el aspecto mejor apreciado fue la satisfacción con la información recibida durante su proceso $(M=4,45, D T=0,50)$. Por último, la utilidad de la formación recibida es considerada alta $(M=4,59, D T=0,58)$, si tienen oportunidad continuarán formándose ( $M 4,09, D T=1,25)$, y entienden que la formación recibida les ayudará a su desarrollo como personas $(M=4,55, D T=0,66)$.

Entre las observaciones cualitativas extraídas de los cuestionarios puede leerse: "buenas profesoras y bastante atentas hacia el alumnado, ya sea personalmente o académicamente" [alumna, 18 años, Proyecto Joven], "me ha gustado mucho y he aprendido y cogido mucha confianza con los profesores" [alumno, 16 años, Es Pinaret], "es un buen profesor, es muy preparado y muy buena persona" [alumno, 16 años, Es Pinaret].

Del cuestionario de satisfacción de los padres administrado por la Fundación $\mathrm{S}^{\prime}$ Estel se recibieron 43 respuestas. A la pregunta de si conocían los objetivos relacionados con el PIE de su hijo, el 69,77\% contestó que sí, mientras que el $27,91 \%$ respondió que no. Aquellos que contestaron que sí, valoran en su mayoría positivamente el grado de cumplimiento $(M=3,9, D T=1,09)$.

A la pregunta de si creían que su hijo había recuperado las ganas de estudiar o trabajar, y si consideraban que había conseguido integrarse al mundo escolar o al laboral, contestó afirmativamente el 69,77\%, mientras que el 13,95\% respondió que no.

Dentro de los comentarios incluidos en los cuestionarios, pueden leerse: "Gracias por ayudar y educar a nuestro hijo" (febrero, 2018); "Muy agradecida de que mi hijo vuelva a estudiar" (mayo, 2018).

\section{Conclusiones}

El programa escolar que se desarrolla en el IES Can Balo demuestra que alcanzar el éxito desde una perspectiva escolar ordinaria es posible. Este programa presenta varias implicaciones tanto para la educación y la formación integral de los menores/jóvenes infractores con medidas judiciales de privación de libertad como para la educación en general:

» El paradigma de la educación inclusiva ofrece el marco general de actuación, aplicable a todos los ámbitos y estructuras educativas, donde todos los alumnos sin excepción, sean cuales sean sus características y circunstancias, pueden aprender y formarse en un contexto ordinario, siempre que haya una adecuación didáctica y metodológica.

» Implementar este modelo de educación inclusiva supone modificar la cultura, la política y las prácticas docentes de los centros, dejar de ver en el alumno y en sus circunstancias el problema 
principal de su fracaso académico, y fijarse en las oportunidades y mejoras que puede y debe ofrecer el contexto a todo el alumnado para favorecer y posibilitar tanto su aprendizaje académico como su desarrollo de habilidades sociales.

» La educación inclusiva no es una modificación de la educación especial, pensada para las personas con discapacidad, sino que hace referencia a todo el alumnado y a todos los colectivos que, por razones diversas, han sido excluidos o apartados del sistema educativo ordinario, como en el caso particular del alumnado infractor.

» Este programa demuestra cómo un diseño basado en los principios de la educación inclusiva puede lograr el desarrollo integral de los menores que han cometido delitos por los que han sido apartados tempranamente del sistema educativo ordinario, y huye de los programas psicológicos elaborados para dar una respuesta terapéutica centrada única y exclusivamente en el alumno y en sus limitaciones con objeto de asimilarlos a la normalidad.

»El mundo educativo tiene que abandonar los condicionantes asistenciales, terapéuticos y compensatorios, que lo sitúan en la perspectiva del fracaso personal, y abrirse a propuestas pedagógicas y didácticas que tengan una visión relativa del éxito educativo adecuada a cada uno de los alumnos para empoderarlos hacia posturas de autoestima y empatía hacia su propio proceso integral de formación.

»El término éxito debe ser interpretado desde una perspectiva global que abrace más que el significado relacionado con el concepto de rendimiento académico. No solo como la inserción exitosa de los menores que promocionan una etapa educativa, sino como personas que finalizan victoriosamente una fase escolar y personal que promueve su reincorporación educativa y social como personas competentes.

» Resulta indispensable que los profesionales que trabajan en este ámbito estén formados para fortalecer la creación de entornos educativos abiertos y flexibles que prevengan situaciones de comportamiento antisocial, y ofrecer un entorno seguro y rico para el desarrollo de todas las personas. Estos profesionales deben tener un gran compromiso y responsabilidad ético-pedagógica, como explica Jordán (2015).

» Por último, cabe destacar que este programa ha dado lugar a valoraciones muy positivas tanto por parte del alumnado como de sus familias, puesto que representa un cambio en la tendencia formativa y académica de estos menores, al pasar de ser alumnos problemáticos o no escolarizados a ser alumnos de éxito, reconocidos en su proceso formativo académico y personal. 


\section{Referencias}

Ainscow, M. (1995). Necesidades especiales en el aula. Unesco-Narcea.

Ainscow, M., Booth, T. y Dyson, A. (2006). Improving schools, developing inclusion. Routledge.

Ainscow, M. y Messiou, K. (2018). Engaging with the views of students to promote inclusion in education. Journal of Educational Change, 19, 1-17. https://doi.org/10.1007/s10833-017-9312-1

De Andrés, P. y Fernández-Hawrylak, M. (2015). Éxito escolar en Educación Básica: Trabas y posibles soluciones. En S. Rodríguez, M. J. López y T. Ambrona (Eds.), Diferentes perspectivas de la educación del siglo xxı, cap. x (pp. 125-139). Asire Educación-Diputación Provincial de Burgos.

Arnaiz, P. (2019). La educación inclusiva en el siglo xxI: Avances y desafíos. Servicio de Publicaciones de la Universidad de Murcia.

Barton, L. (2009). Estudios sobre discapacidad y la búsqueda de la inclusividad: Observaciones. Revista de Educación, 349, 137-152.

Blanco, R. (2008). Marco conceptual sobre educación inclusiva [taller]. 48. ${ }^{a}$ reunión de la Conferencia Internacional de Educación "La educación inclusiva: el camino hacia el futuro" (pp. 5-14). Ginebra. 25 a 28 de noviembre del 2008. http://www.ibe.unesco.org/fileadmin/ user_upload/Policy_Dialogue/48th_ICE/CONFINTED_48_Inf_2__ Spanish.pdf

Booth, T. y Ainscow, M. (2002). Index for inclusion: Developing learning and participation in schools. Centre for Studies on Inclusive Education(CSIE).https://www.eenet.org.uk/resources/docs/Index\%20 English.pdf

Decreto por el cual se regula la atención a la diversidad y la orientación educativa en los centros educativos no universitarios sostenidos con fondos públicos (Decreto 39/2011, 29 de abril). Boletín Oficial de las Islas Baleares, 67. http://boib.caib.es/pdf/2011067/mp97.pdf (Corrección de errores: вOІв, 78. http://boib.caib.es/pdf/2011078/mp61.pdf).

Decreto por el que se crea el IES Can Balo, en el Pont d'Inca, en el término municipal de Marratxí (Decreto 25/2017, 19 de mayo). Boletín Oficial de las Islas Baleares, 62. http://www.caib.es/eboibfront/pdf/ es/2017/62/979397

Decreto por el que se establece el currículo de la Educación Secundaria Obligatoria en las Islas Baleares (Decreto 34/2015, 15 de mayo). Boletín Oficial de las Islas Baleares, núm. 73. http://www.caib.es/eboibfront/pdf/es/2015/73/919862

Echeita, G. (2019). Educación inclusiva: El sueño de una noche de verano. Octaedro. 
Echeita, G. y Ainscow, M. (2011). Educación inclusiva como derecho: Marco de referencia y pautas de actuación para el desarrollo de una revolución pendiente. Tejuelo, 12, 26-46.

Echeita, G., Simón, C., Verdugo, M. A., Sandoval, M., López, M., Calvo, I. y González, F. (2009). Paradojas y dilemas en el proceso de inclusión educativa en España. Revista de Educación, 349, 153-178.

Edel, R. (2003). El rendimiento académico: Concepto, investigación y desarrollo. Revista Iberoamericana sobre Calidad, Eficacia y Cambio en Educación, Reice, 1(2), 1-15.

Fandiño, Y. J. (2008). Una enseñanza e investigación inteligentes de la inteligencia para el éxito escolar y el éxito en la vida cotidiana. Revista Iberoamericana de Educación 46(9), 1-12. https://doi.org/10.35362/ rie4691881

Feito, R. (2009). Éxito escolar para todos. Revista Iberoamericana de Educación, 50, 131-151. https://doi.org/10.35362/rie500666

Fernández-Campoy, J. M., Aguilar-Parra, J. M., Domínguez, J. C, Rueda-Rosas, P. y Lozano, M. C. (2017). La relevancia de los programas educativos y formativos como elementos favorecedores del proceso de reinserción social y laboral de los menores infractores. International Journal of Developmental and Educational Psychology, 2(1), 405-415. https://doi.org/10.17060/ijodaep.2017.n1.v2.953

García-Poole, C., Byrne, S. y Rodrigo, M. J. (2019). How do communities intervene with adolescents at psychosocial risk? A systematic review of positive development programs. Children and Youth Services Review, 99, 194-209. https://doi.org/10.1016/j.childyouth.2019.01.038

Gimeno, J. (2000). La educación obligatoria: Su sentido educativo y social. Morata.

Instrucción del Director General de Innovación y Comunidad Educativa por la que se regula el funcionamiento del IEs Can Balo en los centros docentes sostenidos con fondos públicos durante el curso 20182019 (Instrucción 20/18, 1. ${ }^{\circ}$ de septiembre).

Jordán, J. A. (2015). La responsabilidad ética-pedagógica de los profesores-educadores: Una mirada nueva desde Max van Manen. Revista Española de Pedagogía, 261, 381-396.

Kremer, K. P., Maynard, B. R., Polanin, J. R., Vaughn, M. G. y Sarteschi, C. M. (2015). Effects of after-school programs with at-risk youth on attendance and externalizing behaviors: A systematic review and meta-analysis. Journal of Youth and Adolescence, 44(3), 616-636. https://doi.org/10.1007/s10964-014-0226-4

Ley Orgánica para la Mejora de la Calidad Educativa (Lomce) (Ley Orgánica 8/2013, 9 de diciembre). Boletín Oficial del Estado, 295, 2013, 10 de diciembre. https:/www.boe.es/eli/es/lo/2013/12/09/8/con 
Ley Orgánica reguladora de la Responsabilidad Penal de los Menores (LORPM) (Ley Orgánica 5/2000, 12 de enero). Boletín Oficial del Estado, 11. https://www.boe.es/eli/es/lo/2000/01/12/5/con

López, N., Fernández-Hawrylak, M., Soldevila, J. y Muntaner, J. J. (2018). El trabajo con familias de menores infractores: Elemento clave en el proceso inclusivo. Revista Aula Abierta de la Universidad de Oviedo, 47(2), 159-166. https://doi.org/10.17811/rifie.47.2.2018.159-166

Luna, E., Monllaó, E., Palou, B., Panchón, C., Sandín, M. P. y Tapias, M. A. (2018). L'aprenentatge servei com a innovació metodològica a medi obert. Generalitat de Catalunya, Centre d'Estudis Jurídics i Formació Especialitzada.

Mampaso, J., Pérez, F., Corbí, B., González, M. P. y Bernabé, B. (2014). Factores de riesgo y de protección en menores infractores: Análisis y prospectiva. Psychologia Latina, 5(1), 11-20.

Marchesi, Á. (2014). Del lenguaje de la deficiencia a las escuelas inclusivas. En C. Coll, Á. Marchesi y J. Palacios (Coords.), Desarrollo psicológico y educación: III Trastornos del desarrollo y necesidades educativas especiales (pp. 21-43). Alianza.

Muntaner, J. J., Rosselló, M. R. y De la Iglesia, B. (2016). Buenas prácticas en educación inclusiva. Educatio Siglo Xxı, 34(1), 31-50. https://doi. org/10.6018/j/252521

Organización de las Naciones Unidas. (1990, 14 de diciembre). Resolución 45/112 de la Asamblea General de las Naciones Unidas, "Directrices de las Naciones Unidas para la prevención de la delincuencia juvenil (Directrices de Riad)". https://www.ohchr.org/sp/ professionalinterest/pages/preventionofjuveniledelinquency.aspx

Organización de las Naciones Unidas para la Educación, la Ciencia y la Cultura (Unesco). (2016). Educación 2030. Declaración de Incheon: Hacia una educación inclusiva y equitativa de calidad y un aprendizaje a lo largo de la vida para todos. https://unesdoc.unesco.org/ ark:/48223/pf0000245656_spa

Organización de las Naciones Unidas para la Educación, la Ciencia y la Cultura (Unesco). (2017). Guía para asegurar la inclusión y la equidad en la educación. https://unesdoc.unesco.org/ark:/48223/pf0000259592

Palacios, A. y Romañach, J. (2006). El modelo de la diversidad: La bioética y los derechos humanos como herramienta para alcanzar la plena dignidad en la diversidad funcional. Diversitas.

Parrilla, Á. (2002). Acerca del origen y sentido de la educación inclusiva. Revista de Educación, 327, 11-29.

Pérez, A. (2017). Model d'intervenció socioeducativa per millorar el rendiment acadèmic dels adolescents tutelats i residents en centres de protecció. Butlletí d'Inf@ncia, 102. 
Poch, R. y Zaplana, T. (2017). Factors protectors i de risc que incideixen en les trajectòries educatives dels menors i joves interns als Centres Educatius de Justícia catalans. Generalitat de Catalunya, Centre d'Estudis Jurídics i Formació Especialitzada.

Pujolàs, P. (2008). 9 Ideas clave: El aprendizaje cooperativo. Graó.

Pujolás, P., Lago, J. R. y Naranjo, M. (2013). Aprendizaje cooperativo y apoyo a la mejora de las prácticas inclusivas. Revista de Investigación en Educación, 11(3), 207-218. Sapon-Shevin, M. (2013). La inclusión real: Una perspectiva de justicia social. Revista de Investigación en Educación, 11(3), 71-85.

Stainback, S., Stainback, W. y Moravec, J. (1999). Un currículo para crear aulas inclusivas. En S. Stainback y W. Stainback (Coords.), Aulas inclusivas (pp. 83-102). Narcea.

Wehmeyer, M. L. (2009). Autodeterminación y la tercera generación de prácticas de inclusión. Revista de Educación, 349, 45-67. 